\title{
Efeitos do Extrato Hidroetanólico de Simaba ferruginea St. Hil (calunga) (EHSF) no Ciclo Estral e Acasalamento de Ratas Wistar: Consequência no Desenvolvimento Intrauterino da Prole \\ Effects of the Hydroethanolic Extract of Simaba Ferruginea St. Hil (calunga) (EHSF) on the Estrous Cycle and Mating of Wistar Rats: Consequence in the Intrauterine Development of the Offspring
}

\author{
Maria Luzinete Alves Vanzeler*a; Wander Miguel de Barros ${ }^{\mathrm{b}}$; Antônia Gladys Nasello ${ }^{\mathrm{c}}$ \\ ${ }^{a}$ Universidade Federal de Mato Grosso, Faculdade de Medicina. MT, Brasil. \\ 'Instituto Federal de Educação, Ciência e Tecnologia de Mato Grosso. MT, Brasil. \\ ${ }^{c}$ Faculdade de Ciências Médicas da Santa Casa de São Paulo. SP, Brasil. \\ *E-mail:van.zeler@hotmail.com
}

\begin{abstract}
Resumo
O rizoma da Simaba ferruginea St. Hil tem sido utilizado, popularmente, para úlceras, febres, diarreias e processos inflamatórios. Estudos farmacológicos têm ratificado algumas destas ações. Entretanto, os efeitos na reprodução não estão elucidados. O objetivo deste trabalho foi estudar efeitos do extrato hidroetanólico $(70 \%)$ do rizoma da Simaba ferruginea St. Hil (EHSF) na reprodução de ratas Wistar e conseqüências desses efeitos no desenvolvimento intra-uterino da prole. Para tanto, os animais foram tratados com EHSF 50 e $100 \mathrm{mg} \mathrm{Kg}^{-1} \mathrm{ou}_{\text {água destilada }}$ por 15 dias e verificaram-se os efeitos no ciclo estral, acasalamento, número de fetos no útero, peso úmido do útero/ovário, consumo de água e ração, peso de outros órgãos internos e nos parâmetros bioquímicos do soro. O estudo do ciclo estral mostrou que $100 \%$ das ratas completaram o ciclo, e não foram observadas alterações no ganho de peso corporal, consumo de água e ração, peso úmido do coração, fígado, pulmões, baço, rins e útero/ovário; porém, a concentração da aspartato aminotransferase (AST) foi aumentada. A implantação dos fetos foi reduzida com a maior dose. Estes dados sugerem que o EHSF pode apresentar hepatotoxicidade, e que a redução no número de fetos implantados não está relacionada com interferência no ciclo estral nem no acasalamento.
\end{abstract}

Palavras-chave: Simaba Ferruginea. Reprodução. Ratas. Prole. Toxicidade

\begin{abstract}
The rhizome of Simaba ferruginea St. Hil has been popularly used in the treatment of ulcers, fever, diarrhea and inflammatory processes. Pharmacological studies have ratified some of these actions. However, the effects on reproduction have not been elucidated. This study aimed to study the effects of the hydroethanolic extract (70\%) from the rhizome of Simaba ferruginea St. Hil (EHSF) on the reproduction of Wistar rats and the consequences on the intrauterine development of offspring. For this purpose, the animals were treated with EHSF 50 and $10 \mathrm{mg}$ $\mathrm{Kg}^{-1}$ or vehicle for 15 days and effects on the estrous cycle, mating, amount of fetuses in the uterus, uterus / ovary fresh weight, consumption of water and food, weight of the other internal organs and biochemical parameters of the serum were observed. The study of the estrous cycle showed that $100 \%$ of the rats preserved the cycling sequence, and alterations on the body weight gain, consumption of water and food, fresh weight of heart, liver, lungs, spleen, kidneys and uterus / ovary were not observed; however, the concentration of aspartate aminotransferase (AST) was increased. The implantation of fetuses was decreased with administration of the highest dose. This data suggest that EHSF may increase hepatoxicity, and that the reduction on the amount of implanted fetuses is not related with the interference on neither estrous cycle nor mating. Further studies are necessary in order to explain this data.
\end{abstract}

Keywords: Simaba ferruginea. Reproduction. Rats. Offspring. Toxicity.

\section{Introdução}

A milhares de anos as plantas medicinais vêm sendo utilizadas como recurso terapêutico em todo o mundo (ARNOUS et al., 2005). Muitos países, hoje em dia só permitem o uso de fitoterápicos com prescrição médica (MARCONATO et al., 2019).

No Brasil o primeiro fitoterápico foi registrado em 1929, na primeira edição da Farmacopeia Brasileira (BRANDÃO et al., 2006). Várias décadas se passaram e ainda hoje os fitoterápicos sofrem restrições quanto ao uso e aceitação, devido ao reduzido número de estudos que comprovam a ação biológica e segurança quanto a efeitos tóxicos agudos, crônicos e em especial a toxicidade reprodutiva (SHARAPIN, 1999; SONAGLIO, 1987).

Em relação a reprodução, há grande necessidade de estudos para determinação dos potenciais tóxicos. Especialmente quanto a toxicidade durante a gestação, quando pode ocorrer alterações fetais decorrentes da exposição da mãe a agentes químicos, efeitos abortivos, teratogênicos e citotóxicos (SGUAREZI et al., 2017)

A maior parte dos fitoterápicos que são utilizados atualmente não tem o perfil toxicológico bem conhecido (CAPASSO et al., 2000; VEIGA-JUNIOR e MELO, 2008) e são raros os trabalhos relacionados a efeitos sobre reprodução e desenvolvimento. Evidências científicas de ocorrência de intoxicações e efeitos colaterais relacionados com o uso de plantas medicinais e fitoterápicos constituem-se em informações que dificilmente chegam ao alcance dos usuários atendidos nos serviços públicos de saúde (ALEXANDRE et al., 2008; SILVA et al., 2006; SILVEIRA, BANDEIRA e 


\section{ARRAIS, 2008).}

Os produtos fitoterápicos de acordo com a legislação brasileira de toxicidade reprodutiva devem comprovar a eficácia e a segurança, através da realização de avaliação de efeitos sobre a performance reprodutiva, o que envolve critérios de infertilidade e efeitos adversos no decorrer da vida intra e extrauterina, efeitos adversos sobre a gestante, durante o parto e acompanhamento do desenvolvimento pós-natal (CARVALHO et al., 2008).

A Simaba ferruginea St. Hil (Simaroubaceae), conhecida como calunga ou fel-da-terra, é planta nativa do cerrado Matogrossense, utilizada popularmente para o tratamento de úlceras gástricas, febres, diarreias, dores e inflamações (LORENZI, 2002; MARCELLO, 2001). Estudos farmacológicos evidenciaram os efeitos anti-ulcerogênicos nos modelos de indução por etanol e indometacina (MARCELLO, 2001); atividade anti-úlcerogênica e anti-nociceptiva (NOLDIN, 2005); e atividade antimicrobiana (SILVA JUNIOR, 2009); Em relação a reprodução foi verificado redução do número de fetos nas fases gestacionais de organogênese e período fetal, bem como redução no número de descendentes nascidos vivos (VANZELER et al., 2015). Porém faltam relatos de atividades toxicológicas sobre o ciclo estral, fertilidade e desempenho reprodutivo (acasalamento e prenhez). O presente trabalho objetivou avaliar o efeito do extrato hidroetanólico de Simaba ferruginea St. Hil (calunga) (EHSF) no ciclo estral, fertilidade e desempenho reprodutivo em ratas Wistar, bem como verificar efeitos tóxicos sobre órgãos internos destes animais.

\section{Material e Métodos}

\subsection{Coleta e identificação da planta}

Foram coletados rizomas de Simaba ferruginea St. Hil no dia 14 de fevereiro de 2004, na Fazenda Experimental da Universidade Federal de Mato Grosso (UFMT) localizada em Santo Antônio do Leverger - MT, a $30 \mathrm{Km}$ ao sul de Cuiabá MT em altitude de $141 \mathrm{~m}$; latitude de $15^{\circ} 47^{\prime} 11^{\prime \prime} \mathrm{S}$ e longitude de $56^{\circ} 04^{\prime} 47^{\prime \prime} \mathrm{W}$.

A identificação taxonômica foi realizada pelo Prof. Dr. Germano Guarim Neto, do Departamento de Botânica e Ecologia do Instituto de Biociências da Universidade Federal de Mato Grosso. As amostras testemunhas do material florífero e herborizado encontram-se depositadas no Herbário Central da Universidade Federal de Mato Grosso, (exsicata no 22591).

\subsection{Preparo do extrato}

Os rizomas de Simaba ferruginea St. Hil foram limpos, secos à temperatura ambiente e triturados em moinho elétrico. O pó resultante foi pesado e macerado em solução hidroetanólica a 70\% (v/v), na proporção de $1: 3(\mathrm{p} / \mathrm{v})$, por 7 dias. Após esse período, os macerados foram filtrados em papel de filtro (n⿳⺈ $170 \mathrm{~g})$. O filtrado foi submetido à rotaevaporação, a $45^{\circ} \pm 1^{\circ} \mathrm{C}$, sob pressão reduzida ( $625 \mathrm{mmHg}$ ), até obtenções dos extratos fluídos. Estes foram postos em estufa a $45^{\circ} \mathrm{C}$, até a completa eliminação do solvente. Após a eliminação do solvente o extrato hidroetanólico de Simaba ferruginea St. Hil (EHSF) foi armazenado em fraco âmbar e mantido vedado dentro de geladeira a $4 \pm 2{ }^{\circ} \mathrm{C}$.

\subsection{Animais}

As ratas utilizadas no experimento são albinas e pertencem a espécie Rattus norvegicus, linhagem Wistar, adultas com aproximadamente 2 meses de idade, pesando entre 200 e 250 gramas. Todas provenientes do Biotério Central da Universidade Federal de Mato grosso (UFMT). Elas eram mantidas em gaiolas de polipropileno a $22 \pm 1{ }^{\circ} \mathrm{C}$, em ciclo claro-escuro de $12 \mathrm{~h}$ (de 7:00h às 19:00h), controlado por "timer" da marca Fox Lux. Os animais tinham acesso livre a água e ração Purina ${ }^{\circledR}$ (Labina).

\subsection{Comissão de ética em experimentação animal}

Este trabalho foi avaliado pela Comissão de Ética da Faculdade de Ciências Médicas da Santa Casa de São Paulo (CEEA), (protocolo no 123, 2006). Os estudos foram realizados de acordo com as normas do Código de Ética Profissional do Médico Veterinário e do Colégio Brasileiro de Experimentação Animal (COBEA).

\subsection{Efeitos do EHSF no ciclo estral de ratas}

Para este procedimento foram selecionadas 36 ratas de aproximadamente 02 meses de idade, virgens, sadias e ciclando normalmente por 03 ciclos consecutivos. Estes animais foram randomicamente divididos em 03 grupos e tratados por 15 dias do seguinte modo: grupo 1- tratadas com EHSF $50 \mathrm{mg} \mathrm{Kg}^{-1}$; grupo 2- tratadas com EHSF $100 \mathrm{mg} \mathrm{Kg}^{-1}$; grupo 3 (controle) - tratadas com água destilada por gavagem orogástrica. O ciclo foi acompanhado através de observações microscópicas diárias do esfregaço vaginal. Os esfregaços foram feitos sempre entre $10 \mathrm{~h}$ e $12 \mathrm{~h}$ da manhã, através da aplicação de $0,5 \mathrm{~mL}$ de solução de $\mathrm{NaCl} 0,9 \%$ na vagina, com uma pipeta de plástico de $1 \mathrm{~mL}$, seguida de aspiração do lavado. O material pipetado era depositado sobre uma lâmina e levado ao microscópio ótico e observados as fases do ciclo estral, segundo Hankness e Wagner (1993) e Barril et al., (2016). As ratas deste experimento foram pesadas a cada 02 dias e ao final do período de tratamentos, foram sacrificadas e o soro coletado, para análise de alguns parâmetros bioquímicos.

\subsection{Efeitos do EHSF na fertilidade e desempenho reprodutivo de ratas}

Trinta ratas ciclando normalmente por 3 ciclos consecutivos foram tratadas por 15 dias com EHSF do seguinte modo: 10 animais com a dose de $50 \mathrm{mg} \mathrm{Kg}^{-1}, 10$ animais com $100 \mathrm{mg} \mathrm{Kg}^{-1}$, e 10 animais com água destilada (grupo controle). Durante o período de tratamento o ciclo estral foi acompanhado diariamente, e no $15^{\circ}$ dia as ratas foram acasaladas. Após o acasalamento o lavado vaginal 
continuou sendo observado diariamente até o aparecimento de espermatozóide e / ou tampão mucoso (1ำ dia de prenhes). No $10^{\circ}$ dia de prenhes, todas as ratas foram sacrificadas com éter etílico e feita a laparotomia exploratória, após este procedimento, foram retirados e pesados o útero e ovários, contados os fetos e verificado a presença ou ausência de reabsorção fetal.

\subsection{Análise estatística}

Os resultados estão expressos em Média \pm Erro Padrão e a diferença entre os grupos foram verificados através do teste de Análise de Variância (ANOVA), seguido pelo teste de Dunnett's ou de Student-Newman-Kelus, sendo o nível de significância estatística considerado $(\mathrm{p}<0,05)$.

\section{Resultados e Discussão}

O Quadro 1 mostra que $100 \%$ das ratas controle e tratadas com EHSF (50 e $100 \mathrm{mg} \mathrm{Kg}^{-1}$ ) ciclaram normalmente durante os 15 dias de tratamento, e não houve alteração estatisticamente significante na duração do ciclo (teste de ANOVA).

Quadro 1 - Influência da administração do extrato hidroetanólico de Simaba ferruginea St. Hil por gavagem orogástrica, durante 15 dias, no ciclo estral de ratas

\begin{tabular}{|c|c|c|c|}
\hline \multirow{2}{*}{ Tratamentos } & \multirow{2No}{*}{} & \multicolumn{2}{|c|}{ Ciclo Estral } \\
\cline { 3 - 4 } & $\begin{array}{c}\text { \% de Ratas que } \\
\text { Ciclaram }\end{array}$ & $\begin{array}{c}\text { Duração em } \\
\text { Dias } \\
\text { (Média } \pm \text { EP) }\end{array}$ \\
\hline Água destilada & 12 & 100 & $4,6 \pm 0,1$ \\
\hline EHSF $50 \mathrm{mg} \mathrm{Kg}^{-1}$ & 12 & 100 & $4,2 \pm 0,1$ \\
\hline EHSF $100 \mathrm{mg} \mathrm{Kg}^{-1}$ & 12 & 100 & $3,9 \pm 0,1$ \\
\hline
\end{tabular}

Teste estatístico ANOVA, uma via; EP (Erro Padrão); № = número de animais

Fonte: Dados da pesquisa.

O Quadro 2 mostra que o Pré-tratamento por 15 dias de ratas com EHSF $100 \mathrm{mg} \mathrm{Kg}^{-1}$ provocou redução no número de fetos implantados $(7,9 \pm 3,3)$ em comparação ao grupo controle $(10,7 \pm 1,5)(\mathrm{p}<0,05$ Teste de ANOVA seguido de Dunett $) ;$ já a dose de $50 \mathrm{mg} \mathrm{Kg}^{-1}$ não provocou diferença estatisticamente significante no número de fetos implantados. Ainda foi verificado que $100 \%$ das ratas procederam acasalamento.

Quadro 2 - Número de ratas que acasalaram e de fetos implantados após pré-tratamento por 15 dias com Simaba ferruginea St. Hil por gavagem orogástrica. Dados em Média \pm Erro Padrão

\begin{tabular}{|c|c|c|c|}
\hline Tratamento & № & $\begin{array}{c}\text { \% de fêmeas } \\
\text { que acasalaram }\end{array}$ & $\begin{array}{c}\text { Número de fetos } \\
\text { vivos }\end{array}$ \\
\hline Água destilada & 10 & 100 & $10,7 \pm 1,5$ \\
\hline EHSF $50 \mathrm{mg} \mathrm{Kg}^{-1}$ & 10 & 100 & $9,0 \pm 2,7$ \\
\hline EHSF $100 \mathrm{mg} \mathrm{Kg}^{-1}$ & 10 & 100 & $7,9 \pm 3,3^{*}$ \\
\hline
\end{tabular}

* $\mathrm{p}<0,05$, em relação ao grupo controle; teste estatístico de ANOVA uma via, seguido do teste de Dunett; № = número de animais por grupo, EHSF extrato de Simaba ferruginea St. Hil

Fonte: Dados da pesquisa.

Quanto ao peso relativo do ovário / útero, não houve alterações, entre os grupos tratados com EHSF $50 \mathrm{mg} \mathrm{Kg}^{-1}$ $(0,011 \pm 0,004)$ e $100 \mathrm{mg} \mathrm{Kg}^{-1}(0,009 \pm 0,005)$ em relação ao grupo controle $(0,01 \pm 0,002)(\mathrm{p}>0,05$ Teste de ANOVA), respectivamente (Quadro 3).

Quadro 3 - Efeito do extrato Simaba ferruginea St. Hil por gavagem orogástrica, durante quinze dias, no peso úmido útero/ ovário de ratas. Dados em Média \pm Erro Padrão

\begin{tabular}{|c|c|c|}
\hline Traramentos & № & Peso úmido útero/ovário (g) \\
\hline Água destilada & 10 & $0,01 \pm 0,002$ \\
\hline EHSF $50 \mathrm{mg} \mathrm{Kg}^{-1}$ & 10 & $0,011 \pm 0,004$ \\
\hline EHSF $100 \mathrm{mg} \mathrm{Kg}^{-1}$ & 10 & $0,009 \pm 0,005$ \\
\hline
\end{tabular}

Teste estatístico = ANOVA uma via; № = número de animais por grupo; EHSF (extrato de Simaba ferruginea St. Hil).

Fonte: Dados da pesquisa

O tratamento com EHSF (50 e $100 \mathrm{mg} \mathrm{Kg}^{-1}$ ) por 15 dias não alterou o consumo de ração, o consumo hídrico nem o peso corporal das ratas quando comparadas ao grupo controle (tratados com água destilada) (teste de ANOVA) (Quadros 4, 5 e 6$)$.

Quadro 4 - Efeito do extrato Simaba ferruginea St Hil. por gavagem orogástrica, durante 15 dias, no consumo de ração de ratas. Dados em Média \pm Erro Padrão

\begin{tabular}{|c|c|c|c|}
\hline \multirow{2}{*}{$\begin{array}{c}\text { Dias de } \\
\text { Tratamento }\end{array}$} & \multicolumn{3}{|c|}{ Consumo de ração (g) } \\
\cline { 2 - 4 } & $\begin{array}{c}\text { EHSF } \\
\text { destilada (12) }\end{array}$ & $\begin{array}{c}\mathbf{5 0} \mathrm{mg} \mathrm{Kg}^{-1} \\
\mathbf{( 1 2 )}\end{array}$ & $\begin{array}{c}\mathbf{1 0 0} \mathrm{mg} \mathrm{Kg}^{-1} \\
\mathbf{( 1 2})\end{array}$ \\
\hline $2^{\circ} \mathrm{o}$ & $21,6 \pm 1,2$ & $19,4 \pm 1.2$ & $18.9 \pm 1.1$ \\
\hline $4^{\circ}$ & $23,5 \pm 0.9$ & $21.3 \pm 0.6$ & $20.5 \pm 1.7$ \\
\hline $6^{\circ}$ & $24,3 \pm 1.1$ & $22.2 \pm 1.8$ & $19.9 \pm 2.1$ \\
\hline $8^{\circ}$ & $24,9 \pm 1.6$ & $23.7 \pm 1.3$ & $21.9 \pm 1.4$ \\
\hline $10^{\circ}$ & $21,8 \pm 0.8$ & $20.6 \pm 0.7$ & $19.8 \pm 0.8$ \\
\hline $12^{\circ}$ & $26,2 \pm 1.6$ & $24.6 \pm 1.7$ & $23.6 \pm 1.8$ \\
\hline $15 \underline{o}$ & $29,4 \pm 2.1$ & $27.5 \pm 0.9$ & $26.4 \pm 0.9$ \\
\hline
\end{tabular}

Teste Estatístico ANOVA; (12) número de animais por grupo; EHSF (extrato de Simaba ferruginea St. Hil)

Fonte: Dados da pesquisa.

Quadro 5 - Efeito do extrato Simaba ferruginea St Hil. por gavagem orogástrica, durante 15 dias, no consumo hídrico de ratas. Dados em Média \pm Erro Padrão

\begin{tabular}{|c|c|c|c|}
\hline \multirow{3}{*}{$\begin{array}{c}\text { Dias de } \\
\text { Tratamento }\end{array}$} & \multicolumn{3}{|c|}{ Consumo hídrico $(\mathrm{mL})$} \\
\hline & \multicolumn{3}{|c|}{ EHSF } \\
\hline & $\begin{array}{c}\text { Água destilada } \\
\text { (12) }\end{array}$ & $\begin{array}{l}50 \mathrm{mg} \mathrm{Kg}^{-1} \\
\text { (12) }\end{array}$ & $\begin{array}{c}100 \mathrm{mg} \mathrm{Kg}^{-1} \\
\text { (12) }\end{array}$ \\
\hline $2^{\mathrm{o}}$ & $42.4 \pm 2.8$ & $41.3 \pm 1.5$ & $38.9 \pm 1.8$ \\
\hline $4^{\circ}$ & $53.5 \pm 1.9$ & $51.3 \pm 2.5$ & $50.5 \pm 2.5$ \\
\hline $6^{0}$ & $54.3 \pm 1.8$ & $52.2 \pm 1.8$ & $49.9 \pm 2.5$ \\
\hline $8^{-}$ & $59.9 \pm 1.6$ & $56.8 \pm 2.3$ & $51.9 \pm 1.8$ \\
\hline $10^{\circ}$ & $61.8 \pm 1.8$ & $60.6 \pm 1.7$ & $59.8 \pm 1.9$ \\
\hline $12^{\mathrm{o}}$ & $66.5 \pm 2.6$ & $64.9 \pm 3.5$ & $63.1 \pm 2.5$ \\
\hline $15^{\circ}$ & $69.4 \pm 5.2$ & $67.5 \pm 2.4$ & $65.4 \pm 3.1$ \\
\hline
\end{tabular}

ANOVA, uma via, (12) número de animais por grupo; veículo = água destilada, EHSF (extrato de Simaba ferruginea St. Hil)

Fonte: Dados da pesquisa. 
Quadro 6 - Influência do extrato Simaba ferruginea St Hil. ou água destilada por gavagem orogástrica, durante 15 dias, no peso corporal de ratas. Dados em Média \pm Erro Padrão

\begin{tabular}{|c|c|c|c|}
\hline \multirow{2}{*}{$\begin{array}{c}\text { Dias de } \\
\text { Tratamento }\end{array}$} & \multicolumn{3}{|c|}{ Peso Corporal (g) } \\
\cline { 2 - 4 } & $\begin{array}{c}\text { EHSF } \\
\text { Água destilada } \\
(\mathbf{1 2})\end{array}$ & $\begin{array}{c}\mathbf{5 0} \mathrm{mg} \mathrm{Kg}^{-1} \\
(\mathbf{1 2})\end{array}$ & $\begin{array}{c}\mathbf{1 0 0} \mathrm{mg} \mathrm{Kg}^{-1} \\
\mathbf{( 1 2 )}\end{array}$ \\
\hline $2^{\circ}$ & $236 \pm 5.9$ & $229 \pm 2.3$ & $223 \pm 2.5$ \\
\hline $4^{\circ}$ & $242 \pm 4.1$ & $234 \pm 3.5$ & $231 \pm 2.9$ \\
\hline $6^{\circ}$ & $238 \pm 4.6$ & $225 \pm 3.7$ & $229 \pm 5.9$ \\
\hline $8^{\circ}$ & $246 \pm 5.3$ & $229 \pm 5.3$ & $222 \pm 2.6$ \\
\hline $10^{\circ}$ & $251 \pm 4.8$ & $238 \pm 2.7$ & $243 \pm 4.9$ \\
\hline $12^{\circ}$ & $248 \pm 3.6$ & $243 \pm 3.8$ & $237 \pm 3.5$ \\
\hline $15^{\circ}$ & $255 \pm 6.2$ & $249 \pm 5.8$ & $239 \pm 3.2$ \\
\hline
\end{tabular}

ANOVA, uma via; (12) número de animais por grupo; EHSF (extrato Simaba ferruginea St. Hil).

Fonte: Dados da pesquisa.

O teste de ANOVA seguido do teste de Student-NewmanKelus, mostrou aumento dos níveis de $\operatorname{ALT}(\mathrm{p}<0,01)$, porém não foi observada alteração estatisticamente significante nos demais parâmetros bioquímicos (Quadro 7).
Quadro 7-Efeito do tratamento com extrato de Simaba ferruginea St Hil. por gavagem orogástrica durante 15 dias na concentração de uréia, transaminase oxalacética e pirúvica, proteínas totais, albumina e glicose. Dados em Média \pm Erro Padrão

\begin{tabular}{|c|c|c|c|}
\hline Medida & \multicolumn{3}{|c|}{ EHSF } \\
\hline & $\begin{array}{c}\text { Veículo } \\
\text { (10) }\end{array}$ & $\begin{array}{c}50 \mathrm{mg} \mathrm{Kg}^{-1} \\
\text { (10) }\end{array}$ & $\begin{array}{c}100 \mathrm{mg} \mathrm{Kg}^{-1} \\
\text { (10) }\end{array}$ \\
\hline Uréia $\mathrm{mg} \mathrm{dL}^{-1}$ & $45 \pm 4,1$ & $48,4 \pm 2,5$ & $38.4 \pm 1,9$ \\
\hline AST (U/I) & $429 \pm 15,3$ & $392,2 \pm 24,3$ & $535,4 \pm 20,4^{*}$ \\
\hline ALT (U/I) & $317,6 \pm 3,7$ & $306,3 \pm 4,2$ & $303,4 \pm 5,3$ \\
\hline $\begin{array}{c}\text { Proteínas } \\
\text { Totais } \mathrm{mg} \mathrm{dL}^{-1}\end{array}$ & $7,5 \pm 0,15$ & $7,7 \pm 0,4$ & $9,6 \pm 0,96$ \\
\hline $\begin{array}{c}\text { Albumina } \\
\mathrm{dL}^{-1}\end{array}$ & $2,6 \pm 0,3$ & $2,8 \pm 0,2$ & $3,4 \pm 0,2$ \\
\hline $\begin{array}{c}\text { Glicose mg } \\
\mathrm{dL}^{-1}\end{array}$ & $110,6 \pm 7,3$ & $99,6 \pm 5,2$ & $105,4 \pm 6,2$ \\
\hline
\end{tabular}

AST $=$ aspartatoaminotranferase; ALT $=$ alaninaaminotransferase; EHSF (extrato de Simaba ferruginea St. Hil); Teste estatístico ANOVA, seguido do teste de Student-Newman-Kelus; ${ }^{*} \mathrm{p}<0,01 ;(10)$ número de animais por grupo Fonte: Dados da pesquisa.

Os pesos relativos do fígado, coração, baço, pulmões e rins, não sofreram alterações estatisticamente significantes pelo tratamento com EHSF (Teste de ANOVA) (Quadro 8).

Quadro 8 - Efeito da administração oral do extrato Simaba ferruginea St Hil, por 15 dias, sobre os pesos relativos de órgãos internos, em ratas (Média \pm Erro Padrão)

\begin{tabular}{|l|c|c|c|c|c|}
\hline \multicolumn{7}{|c|}{ (Peso úmido do órgão / Peso do animal) x 100 (Média \pm EPM) } \\
\hline \multicolumn{1}{|c|}{ Tratamento } & Fígado & Coração & Pulmão & Rim & Baço \\
\hline Água destilada & $3,9 \pm 0,06$ & $0,35 \pm 0,01$ & $0,61 \pm 0,02$ & $0,81 \pm 0,01$ & $0,21 \pm 0,01$ \\
\hline EHSF $50 \mathrm{mg} \mathrm{Kg}^{-1}$ & $4,3 \pm 0,07$ & $0,39 \pm 0,02$ & $0,68 \pm 0,03$ & $0,78 \pm 0,01$ & $0,24 \pm 0,01$ \\
\hline EHSF 100 mg Kg-1 & $4,2 \pm 0,10$ & $0,34 \pm 0,01$ & $0,62 \pm 0,01$ & $0,73 \pm 0,06$ & $0,19 \pm 0,01$ \\
\hline
\end{tabular}

Teste estatístico de ANOVA, uma via, 10 animais por grupo; EHSF (extrato Simaba ferruginea St. Hil).

Fonte: Dados da pesquisa.

O tratamento com EHSF não alterou o ciclo estral (Quadro 1), sugerindo que o extrato não interferiu nas concentrações de hormônios hipotalâmicos-hipofisários, pois é sabido que o ciclo estral normal é dependente de concentrações fisiológicas destes hormônios (DUARTE 2017; MADELLA-OLIVEIRA; QUIRINO; PACHECO, 2014; SARAIVA et al., 2010).

Modificações na produção e liberação de hormônios são identificadas como determinantes no início da fase púbere e entre os hormônios destacadamente importantes estão os estrógenos, as gonadotrofinas e hormônio liberador de gonadotrofinas (ALMEIDA et al., 2013, p.3).

Também foi observado que todas as ratas acasalaram (Quadro 2), mostrando que o EHSF não alterou o comportamento sexual destes animais, isto sugere que os níveis de estrogênios também estavam adequados durante o estro, estando de acordo com Oliveira (2002); Santos et al. (2003), que afirmam que níveis normais de estrogênios são necessários para a aceitação do macho e acasalamento.

A dose de $100 \mathrm{mg} \mathrm{Kg}^{-1}$ de EHSF reduziu o número de fetos presentes na tuba uterina das ratas pré-tratadas por 15 dias (Quadro 2), sugerindo a possibilidade de redução na concentração plasmática de progesterona, pois baixos níveis deste hormônio interferem na viabilidade do embrião
(GALIMBERTI et al., 2001; KATO MORISHIGE; ROTHCHILD, 1979; MARQUES et al., 2013). Por outro lado, o Quadro 3 mostra que o peso do útero/ovário não foi alterado pelo tratamento com EHSF. A determinação do peso do ovário, ainda que seja um processo grosseiro, constitui uma forma indireta de avaliar o estado progestacional materno, pois o maior componente é formado por corpos lúteos que aumentam de volume ao longo da gestação e são as principais fontes de progesterona, conforme Souza, Guerra e Peters (1997); e Vanzeler et al. (2015). Estes dados enfraquecem o argumento de que a redução do número de fetos vivos na tuba uterina (Quadro 2) tenha relação com a redução de progesterona.

Segundo Roblero, Fernández e Croxatto (1987), a sincronia entre o desenvolvimento embrionário, o transporte para o útero e a preparação do endométrio é necessária para a implantação; desta forma, o EHSF poderia estar interferindo com esta sincronia ou interferindo na divisão celular e assim prejudicando a implantação. O extrato ainda poderia estar influenciando a musculatura lisa do útero, pois segundo Brandolt et al. (2007), substâncias que relaxam ou provocam espasmos na musculatura lisa podem interferir no transporte de embriões, dificultando a implantação. 
O EHSF não alterou o consumo de ração (Quadro 4), de água (Quadro 5) e nem o ganho de peso das ratas (Quadro 6). Levando-se em consideração estes parâmetros, o extrato não apresenta toxicidade para ratas adultas, estando de acordo com Chahoud et al. (1999), De Sousa et al. (2013) e Lemônica (2001), que descrevem alterações destes parâmetros como indicativos de toxicidade materna. Por outro lado, o EHSF 100 mg Kg-1 aumentou AST (Quadro 7), indicando possibilidade de hepatotoxicidade, estando de acordo com González e Daza (2003) e Marcello (2001).

Alterações no organismo materno causam alterações do desenvolvimento embrionário, conforme Khera e Hook, (1987). Este autor correlacionou malformações fetais de baixa freqüência como exencefalia, encefalocele, anoftalmia e outras, à redução do peso materno. Embora essa relação tenha sido contestada por Chahoud et al., (1999), não existem dúvidas de que a toxicidade materna pode influenciar no desenvolvimento do embrião. Neste trabalho, observa-se importante dado de toxicidade materna, a redução de ALT. Este poderia estar influenciando indiretamente a fixação ou o desenvolvimento dos fetos no útero.

\section{Conclusão}

Com estes dados, pode ser concluído que o EHSF em doses mais altas pode apresentar hepatotoxicidade, e que a redução no número de fetos implantados não está relacionada com interferência no ciclo estral nem no acasalamento. Outros trabalhos serão necessários para esclarecer estes dados.

\section{Referências}

ALEXANDRE, R.F.; BAGATINI, F.; SIMÕES, C.M.O. Potenciais interações entre fármacos e produtos à base de valeriana ou alho. Rev. Bras. Farmacog., v.18, n.3, p.455-463, 2008. doi: 10.1590/S0102-695X2008000300021

ALMEIDA, O. et al. Endocrinologia da puberdade em fêmeas bovinas. Rev. Cient. Eletr. Med. Vet., v.11, n.20, p.1-13, 2013.

ARNOUS, A.H.; SANTOS, A.S.; BEINNER, R.P.C. Plantas medicinais de uso caseiro-conhecimento popular e interesse por cultivo comunitário. Rev. Espaço Saúde, v.6, n.2, p.1- 6, 2005.

BARRIL, N. et al. Implantação da técnica de citologia vaginal para a identificação e monitorização das fases do ciclo estral em ratas wistar. CuidArte, Enferm, p. 62-164, 2016.

BRANDÃO, M.G.L. et al. Other medicinal plants and botanical products from the first edition of the Brazilian Official Pharmacopoeia. Rev. Bras. Farm., v.18, n.1, p.127-134, 2008. doi: 10.1590/S0102-695X2008000100022.

BRANDOLT, T. D. D. et al. Efeito do extrato de Plectranthus barbatus (Andr.) Benth no desempenho reprodutivo de Rattus novergicus (Berkenhout, 1769). Biotemas, v.20, n.2, p.49-58, 2007. doi: $10.5007 / \% 25 \mathrm{x}$

CAPASSO, R. et al. Phytotherapy and quality of herbal medicines. Fitoterapia, v.71, p. 58-S65, 2000. doi: 10.1016/ s0367-326x(00)00173-8

CARVALHO, A.C.B. et al. Situação do registro de medicamentos fitoterápicos no Brasil. Rev. Bras. Farm., v.18, n.2, p.314-319, 2008. doi: 10.1590/S0102-695X2008000200028.
CHAHOUD, I. et al. Correlation between maternal toxicity and embryo/fetal effects. Reprod. Toxicol., v.13, n.5, p.375-381, 1999. doi: 10.1016/S0890-6238(99)00035-0

DE SOUSA FERREIRA, M.D. et al. Avaliação da toxicidade crônica e gestacional do extrato etanólico de Buchenavia sp., em ratas Wistar. Acta Vet. Bras., v.7, n.4, p.319-326, 2013.

DUARTE, J.C.G. A presença do hormônio concentrator de melatonina no ovário no ciclo reprodutivo de ratas SpragueDawley. São Paulo: Universidade de São Paulo, 2017.

GALIMBERTI, A. M. et al. Taxa de gestação e níveis plasmáticos de progesterona, em receptoras de embrião bovino, tratadas com buserelina após a inovulação. Rev. Bras. Zootec., v.30, n.2, p.353359, 2001. doi: 10.1590/S1516-35982001000200009.

GONZÁLEZ, C.; DAZA, W. Afectación hepatobiliar y pancreática en pacientes con fibrosis quística. Rev. Colomb. Radiol., p.1336-1343, 2003.

HANKNESS, J.E.; WAGNER, J.E. Biologia e clínica de coelhos e roedores. São Paulo: Roca, 1993.

KATO, H.; MORISHIGE, W.K.; ROTHCHILD, I. A quantitative relation between the experimentally determined number of conceptuses and corpus luteum activity in the pregnant rat. Endocrinology, v. 105, n. 3, p. 846-850, 1979. doi: 10.1210/ endo-105-3-846

KHERA, K.S.; HOOK, E.B. Maternal toxicity of drugs and metabolic disorders: a possible etiologic factor in the intrauterine death and congenital malformation: a critique on human data. $C R C$, v.17, n.4, p 345-375, 1987. doi: 10.3109/10408448709029326

LEMÔNICA, I.P. Teratogênese experimental e sua aplicação em humanos. Manual de teratogênese. Porto Alegre: UFRGS, 2001.

LORENZI, H. Árvores brasileiras. Nova Odessa: Plantarum, 2002.

MADELLA-OLIVEIRA, A.F.; QUIRINO, C.R.; PACHECO, A. Principais hormônios que controlam o comportamento reprodutivo e social das fêmeas ruminantes-Revisão. PUBVET, v.8, p.230-339, 2014.

MANSON, J.M.; KANG, Y.J. Test methods for assessing female reproductive and developmental toxicology. In: Principles and methods of toxicology. Raven Press New York, 1994. p.989-1035.

MARCELLO, C.M. Triagem antiúlcera de plantas medicinais usadas popularmente em Mato Grosso para afecções gastrointestinais e validação pré-clínica de Simaba ferruginea St. Hil.(calunga) como antiúlcera. Cuiabá: Universidade Federal de Mato Grosso, 2001.

MARCONATO, A.M. et al. Relação dos Fitoterápicos e Compostos Bioativos na Fertilidade e Infertilidade Humana/List of Phytotherapeutic and Bioactive Compounds in Fertility and Infertility. ID on line Rev. Psicol., v.13, n.44, p.132-139, 2019. doi: 10.14295/idonline.v13i44.1342

MARQUES, T. C. et al. Progesterona no estabelecimento e manutenção da gestação em ruminantes. Enciclopédia Biosfera, Centro Cientifico Conhecer-Goiânia, v. 9, p. 2175-2188, 2013.

NOLDIN, V. F. Estudos fotoquímicos das folhas e rizomas de Simaba ferruginea St. Hil. e avaliação da atividade antiúlcera $e$ antinoceptiva dos extratos e compostos isolados. Itajaí: Universidade do Vale do Itajaí 2005.

OLIVEIRA, S.S. Estudos dos efeitos do Styphnodendron adstringens (Mart) Coville (barbatimão) na reprodução $e$ desenvolvimento intra-uterino e pós-natal de ratos de linhagens Wistar. Cuiabá: Universidade Federal de Mato Grosso, 2003.

ROBLERO, L.S.; FERNÁNDEZ, O.; CROXATTO, H.B. The 
effect of RU486 on transport, development and implantation of mouse embryos. Contraception. v.36 n.5. p.549-555, 1987. doi: 10.1016/0010-7824(87)90007-2

SANTOS, K.R.P. et al. Influência da ausência de luz sobre o ciclo estral de ratas. Arq. Inst. Biol., v.70, n.1 p.21-23, 2003.

SARAIVA, M. V. A. et al. Hormônios hipofisários e seu papel na foliculogênese. Rev. Bras. Reprod. Anim., v.34, p.206-221, 2010.

SGUAREZI, J. G. D. et al. Fitoterápicos na Rede Pública de Saúde (SUS) no Brasil: Um estudo toxicológico de Mikania glomerata em fetos de ratas Wistar. Rev. Fitos, v.10, n.4, p.37598, 2017. doi: 10.5935/2446-4775.20160033

SHARAPIN, N. Medicinal plants: pharmacopoeia prescriptions. Anais da Academia Brasileira de Ciências, v.71, p.295-298, 1999.

SILVA JUNIOR, I.E. et al. Triagem antimicrobiana de algumas plantas medicinais do Cerrado de Mato Grosso. Rev Bras. Farm., v.19, p.242-248, 2009. doi: 10.1590/S0102-695X2009000200011.

SILVA, M.I.G. et al. Utilização de fitoterápicos nas unidades básicas de atenção à saúde da família no município de Maracanaú (CE). Rev. Bras. Farm., v.16, p.455-462, 2006. doi: 10.1590/

\section{S0102-695X2006000400003.}

SILVEIRA, P.F.; BANDEIRA, M.A.M.; ARRAIS, P.S.D. Farmacovigilância e reações adversas às plantas medicinais e fitoterápicos: uma realidade. Rev. Bras. Farm., v.18, n.4, p.61826, 2008. doi: 10.1590/S0102-695X2008000400021.

SONAGLIO, D. Padronização do extrato hidroalcóolico das sumidades floridas de Achyroclines saturcordes (LAM) D. C.Compositae (Marcela). Porto Alegre: Universidade Federal do Rio Grande do Sul, 1987.

SOUZA, E. R.; GUERRA, M. O.; PETERS, V. M. Desenvolvimento de pré-embriões de ratas Wistar da colônia do Biotério do Centro de Biologia da Reprodução. Bol. Centro Biol. Repr., v.16 p.63-70, 1997.

VANZELER, M. L. A. et al. Ensaios preliminares em ratas wistar com extrato hidroetanólico de calunga (Simaba ferruginea St. Hil.) vo, nas fases gestacionais de implantação, organogênese e período fetal: interferências na prole. Rev. Bras. Plantas Med., v.17, n.3, p.454-461, 2015. doi: 10.1590/1983-084X/10_131.

VEIGA-JUNIOR, V. F.; MELLO, J. C. P. As monografias sobre plantas medicinais. Rev. Bras. Farm., v.18 p.464-471, 2008. doi: 10.1590/S0102-695X2008000300022. 